\title{
Optical Response of Metakaolin after Ultraviolet and High Energy Electron Exposure
}

\author{
B. T. Cesul, ${ }^{1}$ S. Mall, ${ }^{1}$ and L. Matson ${ }^{2}$ \\ ${ }^{1}$ Department of Aeronautics and Astronautics, Air Force Institute of Technology, Building 640, \\ 2950 Hobson Way, Wright-Patterson AFB, OH 45433-7765, USA \\ ${ }^{2}$ Metals Branch, Structural Materials Division, Materials and Manufacturing Directorate, Air Force Research Laboratory, \\ 2230 10th Street, Building 655, Wright-Patterson AFB, OH 45433, USA \\ Correspondence should be addressed to S. Mall; Shankar.Mall@afit.edu
}

Received 2 December 2013; Revised 17 March 2014; Accepted 19 March 2014; Published 8 May 2014

Academic Editor: Achim Trampert

Copyright ( $\odot 2014$ B. T. Cesul et al. This is an open access article distributed under the Creative Commons Attribution License, which permits unrestricted use, distribution, and reproduction in any medium, provided the original work is properly cited.

Metakaolin, which is part of a class of inorganic polymers called geopolymers, is being tested currently for its use as a lightweight mirror material in spacecraft applications. Metakaolin, as with most geopolymers, has the advantages of low initial coefficient of thermal expansion, easy preparation at room temperature and pressure, and high specific strength. Even though metakaolin has been known as a structural material for millennia, it has not been properly vetted for use as a material in spacecraft applications, especially with respect to exposure to its environments. This research highlights one particular aspect of response to the space environment; that is, how do the optical properties of metakaolin change after subjugation to bombardment by ultraviolet and high energy electron radiation? These two radiation sources are common in low earth orbit and a primary cause of degradation of organic polymers in space. Photospectroscopic analysis showed that ultraviolet in combination with high energy electrons causes changes in the metakaolin which need to be accounted for due to their potential impacts on the thermal management of a spacecraft and during application in composite mirror structures.

\section{Introduction}

The primary choice of mirror material for spacecraft imaging optics, since the beginning of the space age, has been monolithic glass. Monolithic glass mirrors have enabled spacecraft designers to achieve mirror diameters of over $1 \mathrm{~m}$, and they are well understood in terms of mechanical and thermal performance as monolithic glass variants have been one of the first man-made construction materials [1]. However, material performance requirements for the future space mirrors for advanced imaging missions necessitate a lower areal density than glass with similar if not superior mechanical strength. Additionally, any material chosen must also be able to withstand the unique environment of low earth orbit, namely the near-vacuum conditions, radiation environment and interaction with atomic oxygen.

The space environment poses unique hazards for materials. There is a specific concern with any material, including geopolymers, and it is about the behavior under the radiation environment encountered in orbit. Previous investigations, both on the ground and in-flight experiments, have shown that significant degradation of the organic polymer strength occurs due to the increased cross-linking of polymer networks after absorbing the radiation emitted from the sun or deep space. The typical radiation environment includes exposure to ultraviolet and gamma radiation, in addition to high energy charged particles like electrons and protons [2]. Previous work has shown that for some inorganics, such as siloxane polymers, UV exposure effects were seen as early as after 20-hour exposure [3].

Inorganic polymers, or "geopolymers" (the word being formed from "geologic polymers") as they are commonly known, are generally based on aluminosilicate powders. They have been studied for literally thousands of years as the replacements for traditional cements because of their lower densities and easier curing conditions. Generally, geopolymers have fewer effluent species than their organic counterparts (due to the lack of organic volatiles used in making 
the organic polymers). They cure at lower temperatures than epoxies, reducing the production costs and increasing the categories/choice of materials with which they can be bonded (since the other materials do not have to get exposed to higher temperature environments). Geopolymers have another advantage over the organic polymers in space optics applications; they have a low baseline CTE. The Air Force Research Laboratory, Materials and Manufacturing Directorate has been working on geopolymers over a decade for space optics applications, since geopolymers are promising materials as an adhesive or as a structural material [4].

UV and high energy particle resistance of geopolymers have not been adequately studied under space or simulated space conditions. The most significant related research involving UV exposure of geopolymers has been conducted by the New Jersey Department of Transportation where the UV resilience of a geopolymer coating on a test strip of highway retainer wall was studied [5]. The results from this study indicated that UV was not a factor in geopolymer degradation under ambient atmospheric conditions but recommended further studies under other environmental conditions. Terrestrial UV exposure levels are radically lower than orbital exposure profiles due to its absorption by the Earth's atmosphere. There has not been research on the effects of high energy electron bombardment of geopolymers up to this point as far as the authors are aware.

Therefore, there is a need to determine initial investigation of the performance of metakaolin under radiation conditions as experienced in space environments before these materials can be used in space systems applications. The present paper presents the effects of the space environment on metakaolin, specifically the changes in optical parameter performance after exposure to ultraviolet and high energy electrons. This information is needed before its real-life applications in space optics systems.

\section{Materials and Methods}

2.1. Material and Specimen Details. Metakaolin is a processed form of the kaolinite mineral. The classical chemical formula for kaolinite is $\mathrm{Al}_{2} \mathrm{Si}_{2} \mathrm{O}_{5}(\mathrm{OH})_{4}$. To process kaolinite to metakaolin, endothermic dehydroxylation (i.e., dehydration) is performed by raising the temperature of the kaolinite as high as $550-600^{\circ} \mathrm{C}$ to produce disordered metakaolin, $\mathrm{Al}_{2} \mathrm{Si}_{2} \mathrm{O}_{7}$ [6]. This was done first in the present investigation by applying the direct heat to heat kaolinite in a ceramic pot a fume hood. The metakaolin formulation in the present study was prepared with a 1:2 ratio of metakaolin powder to sodium silicate solution. The sodium silicate trigger solution was a mixture of $26.1 \% \mathrm{SiO}_{2}, 23.9 \% \mathrm{NaOH}$, and $49.9 \% \mathrm{H}_{2} \mathrm{O}$ (percentages by weight). The sodium silicate solution was used to activate the metakaolin geopolymerization reaction, and previous work in the AFRL laboratory had optimized the trigger solution to provide greater cured thermal stability with fewer microcracks on the surface with the stated percentage ratio of $\mathrm{SiO}_{2}$ to $\mathrm{NaOH}$. Postcuring, the $\mathrm{Si}: \mathrm{Al}$ ratio was an average of 2.2 as calculated by XPS data. The BET value was not calculated. After each formulation, the material batches were mixed together in a Thinky ARE250 centrifugal mixer for 2 minutes at $2000 \mathrm{rpm}$. After that, the batches of material were transferred in their slurry form to a blue rubber nitrile mold for curing. The metakaolin mixture initially solidified typically after 40-60 minutes. The partially cured samples were then placed in vacuum sealed bags with pressure on the slurry in mold at an elevated temperature of $60^{\circ} \mathrm{C}$. These were left to solidify overnight. The solidified material was then extracted from the mold and baked under vacuum of $10^{-7}$ Torr vacuum at $140^{\circ} \mathrm{C}$ for 24 hours to drive out internal water molecules that would pose an outgassing risk during space exposure.

2.2. Simulated Space Environment Exposure. Simultaneous UV and high energy electron exposure were performed in Space Combined Effects Primary Test and Research (SCEPTRE) Facility located at the Air Force Research Laboratory. Exposure test in the facility was performed in accordance with the guidelines of ASTM E 512-94, Standard Practice for Combined, Simulated Space Environment Testing of Thermal Control Materials with Electromagnetic and Particulate Radiation, established by the American Society for Testing and Materials [7]. The system has the capability of providing synergistic UV, vacuum ultraviolet (VUV), proton, and electron radiation environments similar to those experienced by satellites orbiting in mid-to-high earth orbits. In addition, the system has the ability to perform in situ measurements of sample temperature and in vacuo reflectance as a function of wavelength. The vacuum level is maintained from approximately $5 \times 10^{-8}$ to $5 \times 10^{-7}$ Torr. The sample temperature ranges from $200^{\circ} \mathrm{C}$ down to $0^{\circ} \mathrm{C}$ depending on its thermooptical properties and the UV intensity averages around three equivalent ultraviolet suns (EUVS) in the $200 \sim 400 \mathrm{~nm}$ wavelength range. The VUV radiation is provided by a Hamamatsu $150 \mathrm{~W}$ deuterium lamp, and it provides approximately 17-EUVS (120 200 nm wavelength range). This combination provides an accelerated testing environment with synergistic effects of vacuum, accelerated UV and electron radiation, and limited thermal cycling [8]. Equivalent solar hours (ESH) are used to correspond the real time test duration to an equivalent exposure time in the orbit.

For the UV exposure test, the SCEPTRE Facility was configured to hold up to eighteen $2.5 \mathrm{~cm}$ diameter disks. The sample's nominal size was $2.5 \mathrm{~cm}$ diameter and $0.3 \mathrm{~cm}$ thickness. These samples were mounted on a central mounting wheel. The samples were placed on the holders using wire braids to keep the samples in place. The sample mounting wheel was rotated to ensure equal exposure to the source over the test period. Each disk was weighed and photographed before and after exposure. There were also unexposed baseline (or control) samples, which were kept outside of the chamber. These were used to compare with the exposed samples. The SCEPTRE chamber details for this test are listed in Table 1. While being in the chamber, the samples were probed periodically for the surface temperature using a handheld IR thermometer through a chamber port window. Video was also taken periodically during the exposure test to record a visual history of the samples. Following the exposure 
TABLE 1: SCEPTRE test conditions.

\begin{tabular}{lc}
\hline Time of exposure & $1096 \mathrm{hrs}$ \\
Solar environment & $2.75 \mathrm{EUVS},(200-400 \mathrm{~nm}) ; 3014 \mathrm{ESH}$ \\
UV source $(2500 \mathrm{~W}$ xenon arc lamp) & $20 \mathrm{EuVS}(115-400 \mathrm{~nm}) ; 2800 \mathrm{ESH}$ \\
VUV source $(150 \mathrm{~W}$ deuterium lamp) & $3 E 9 \mathrm{e}-/ \mathrm{cm}^{2} / \mathrm{sec}$ \\
Electron flux & $6 E 9 \mathrm{e}-/ \mathrm{cm}^{2} / \mathrm{sec}^{2}$ \\
$1 \mathrm{keV}$ electron flux & $3.2 E 16 \mathrm{e}-/ \mathrm{cm}^{2}$ \\
$10 \mathrm{keV}$ electron flux & \\
Electron fluence after exposure time & $75-120^{\circ} \mathrm{C}$ \\
Other exposure test environmental values & $110-160^{\circ} \mathrm{C}$ \\
Inner specimens & $2.3 E-7 \mathrm{Torr}$ \\
Outer specimens & \\
Vacuum pressure & \\
\hline
\end{tabular}

time, the chamber was pumped back to ambient pressure and the samples were extracted.

\section{Results and Discussion}

After SCEPTRE exposure, each sample was immediately weighed, as soon as it was taken out of the chamber while it was still attached to its metal housing. The mass measurement of each sample was taken in similar way before chamber exposure. As seen in Table 2, the mass loss is relatively minimal between samples.

3.1. Photographic and Photometric Measurements. Samples were photographed before and after SCEPTRE exposure with a standard digital camera for visual evidence of color changes and any obvious signs of damage mechanisms. The photometric response of the samples was also taken before and after exposure with a Perkin-Elmer Lambda 950 UV-Vis-NIR double beam spectrophotometer. Photometric signatures were recorded from the UV through IR wavelengths. The signatures in the form of reflectivity measurements for preand postexposure samples were averaged by the wavelength. Since the samples were still mounted to aluminum mounting pucks and held with thin aluminum wire braiding, consistent distances from the sample to the spectrophotometer sensor aperture could not be ascertained that it was consistent. Therefore, the photometric signatures were normalized by setting the peak reflectivity amplitude to unity (1) for each individual sample. So in presenting the data, the curves are shown with reflectivity as a percentage of the maximum reflectivity value for that signature. Since the objective of the study is whether the general behavior of the specimen changed with exposure in terms of its spectral signature, this technique allowed the analysis of the changes. The amplitude of the signature may change as its distance to the sensor changes; that is, it is related to the inverse of the square of distance $\left(1 / r^{2}\right.$ dependence of optical attenuation), but the relative behavior of the individual spectral lines associated with a material will not.

Figure 1 shows the averaged reflectivity values of one of the metakaolin samples at pre- and postexposure to UV. Three curves are shown in Figure 2, which are normalized
TABLE 2: Mass measurements.

\begin{tabular}{lccc}
\hline Sample & $\begin{array}{c}\text { Preexposure } \\
\text { mass }(\mathrm{g})\end{array}$ & $\begin{array}{c}\text { Postexposure } \\
\text { mass }(\mathrm{g})\end{array}$ & \% mass loss \\
\hline Metakaolin 1 & 22.51 & 22.32 & 0.8 \\
Metakaolin 2 & 21.72 & 21.60 & 0.5 \\
Metakaolin 3 & 22.54 & 22.45 & 0.4 \\
Metakaolin 4 & 22.82 & 22.58 & 1.1 \\
\hline
\end{tabular}

reflectivity as the function of wavelength. In this figure, the "Pre, normlzd, avg" curve is the average spectral signature of the pre-UV exposed specimens, normalized for its maximum amplitude signature value. The "Posttest, mounted" line is the average spectral signal of the post-UV exposed specimens while still mounted to the SCEPTRE holding puck with fine metal wire, normalized for its maximum amplitude signature value. The "Posttest, unmounted" line is the average spectral signal of the post-UV exposed specimens unmounted (i.e., just the specimen against the sensor port), normalized for its maximum amplitude signature value. The difference in amplitude between the two post-UV exposure values is attributed to the additional reflectivity added by the highly reflective metal wire holding the specimen in the holding puck. While the post-UV exposure values are not significantly different between the samples with wire and without wire, both results are presented for the sake of completeness.

Figure 1 shows a significant reduction in reflectance over the 200 700 $\mathrm{nm}$ bandwidth (UV through visible) followed by an increased reflectivity in the IR wavelengths. This indicates that the metakaolin was affected by UV and high energy electron exposure. The metakaolin sample's significant change in UV (200 400 $\mathrm{nm})$ and IR (1100-2500 nm) reflectivity is noteworthy. The UV region in Figure 1 shows that after UV exposure, the metakaolin samples became much less reflective to $\mathrm{UV}$, indicating an interesting change that the metakaolin would change from a possible UV reflector to almost definitely UV absorber. In the IR region, a clear transition occurs. In the nonexposed metakaolin, the material begins to lose reflectivity after $900 \mathrm{~nm}$. In the postexposed metakaolin, the reflectivity loss transition point happens at around $1300 \mathrm{~nm}$. The general behavior in terms of peak values 


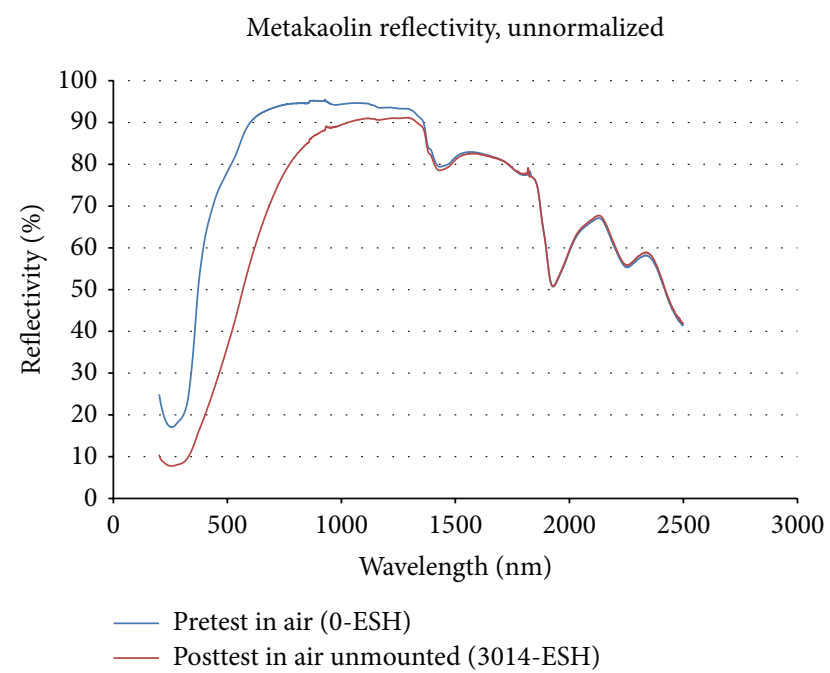

FIGURE 1: Photospectroscopic signature of single metakaolin samples at pre- and postexposure.

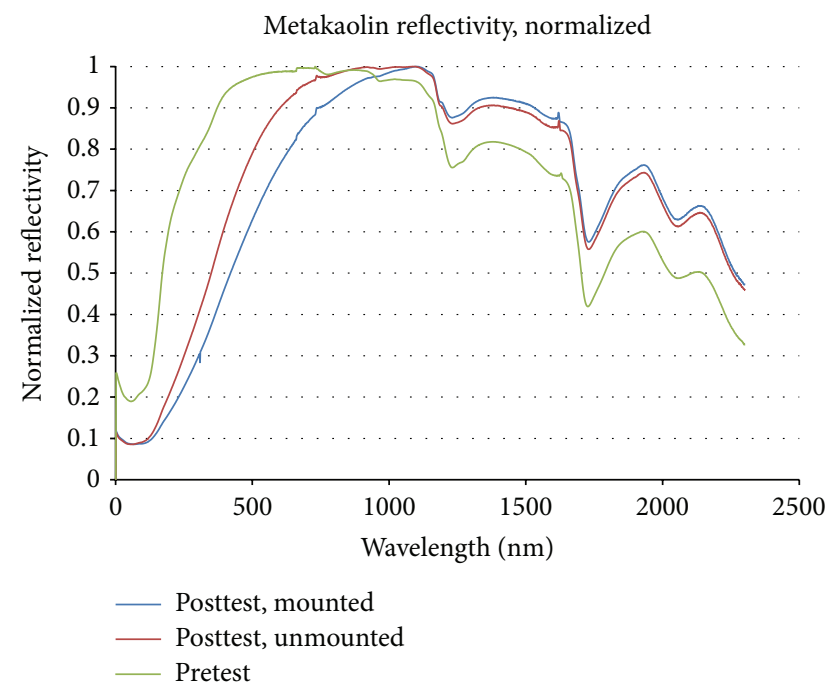

FIGURE 2: Photospectroscopic signature of metakaolin samples at pre- and postexposure, normalized.

is the same following that transition point. However, the shifting to the right of that IR loss transition point and the increase in reflectivity in IR wavelengths following UV exposure of metakaolin may indicate that the metakaolin becomes more of a heat reflector as it ages. It may also be that in the process of UV exposure and high energy particle bombardment, erosion of large surface roughness peaks (at heights greater than IR wavelengths) occurred causing the surface to become smoother and hence more reflective in the IR.

The reduction in reflectivity in the visible wavelengths $(400-700 \mathrm{~nm})$ was consistent with the darkening of the samples as seen in the visible light photographs due to additional oxidation occurring on the surface, typical of many oxide compounds. Reflectance spectroscopy is sensitive to subtle changes in crystal structure or chemistry, and these results seem to confirm a surface chemistry or polymeric structure change following UV and high energy electron exposure. It is also important to note, however, that oxides (which geopolymers are) darken when oxygen atoms are knocked out of the polymer matrix due to UV exposure [9]. This causes vacancies to form in the matrix and these vacancies will refill once exposed to ambient atmosphere with supply of oxygen atoms at room temperature.

\section{Conclusions}

Ultraviolet exposure in combination with high energy electron exposure did affect the metakaolin's optical properties. Photospectroscopic analysis showed a change in behavior in the visible and mid-IR regions. While visible changes were noticeable, they were not unexpected as they are consistent with long understood oxide darkening mechanisms. If left uncoated or untreated and exposed to the space environment, the IR reflectivity changes in the metakaolin may complicate heat management design of a spacecraft.

\section{Conflict of Interests}

The authors declare that there is no conflict of interests regarding the publication of this paper.

\section{Acknowledgments}

The views expressed in this paper are those of the authors and do not reflect the official policy or position of the United States Air Force, Department of Defense, or the U.S. Government. The lead author would like to thank Mr. Cliff Cerbus from the University of Dayton Research Institute who operated and maintained the SCEPTRE Facility. Without his assistance and guidance during the exposure testing, there would have been many more problems without solutions over the time that was available due to paucity of funds. Finally, the authors would also like to acknowledge the support of the Materials and Manufacturing Directorate at WrightPatterson Air Force Base.

\section{References}

[1] P. S. Carlin, "Lightweight mirror systems for spacecraft-an overview of materials \& manufacturing needs," in Proceedings of the IEEE Aerospace Conference, pp. 169-181, Big Sky, Mont, USA, March 2000.

[2] R. A. Dressler, Chemical Dynamics in Extreme Environments, World Scientific, Singapore, 2001.

[3] J. Haffke and J. Woolam, "Synergistic degredation of CV-1144-O due to ultraviolet radiation and heat," in Proceedings of the 6th annual International Conference on Protection of Materials and Structures from Space Environment (ICPMSE '02), pp. 183-191, Toronto, Canada, May 2002.

[4] L. E. Matson, M. Y. Chen, B. deBlonk, and I. Palusinski, "Silicon carbide technologies for lightweighted aerospace mirrors," in Proceedings of the Advanced Maui Optical and Space Surveillance Technologies Conference (AMOSTech '08), Maui, Hawaii, USA, September 2008. 
[5] P. N. Balaguru and M. Arafa, "Geopolymer coating demonstration project for route I-295 scenic overlook," New Jersey Department of Transportation Document FHWA-NJ-2005-021, 2006.

[6] M. Bellotto, A. Gualtieri, G. Artioli, and S. M. Clark, "Kinetic study of the kaolinite-mullite reaction sequence. Part I: kaolinite dehydroxylation," Physics and Chemistry of Minerals, vol. 22, no. 4, pp. 207-217, 1995.

[7] American Society for Testing and Materials, "E 512-94 (Reapproved 2004) Standard practice for combined, simulated space environment testing of thermal control materials with electromagnetic and particulate radiation," ASTM International Document E512-94, 2004.

[8] C. A. Cerbus and P. S. Carlin, "SCEPTRE: the air force combined space environment facility," in Proceedings of the 1st Spacecraft Thermal Control Symposium Proceedings, STCS94USAF Phillips Laboratory, Albuquerque, NM, USA, 1994.

[9] J. Frercks, H. Weber, and G. Wiesenfeldt, "Reception and discovery: the nature of Johann Wilhelm Ritter's invisible rays," Studies in History and Philosophy of Science Part A, vol. 40, no. 2, pp. 143-156, 2009. 

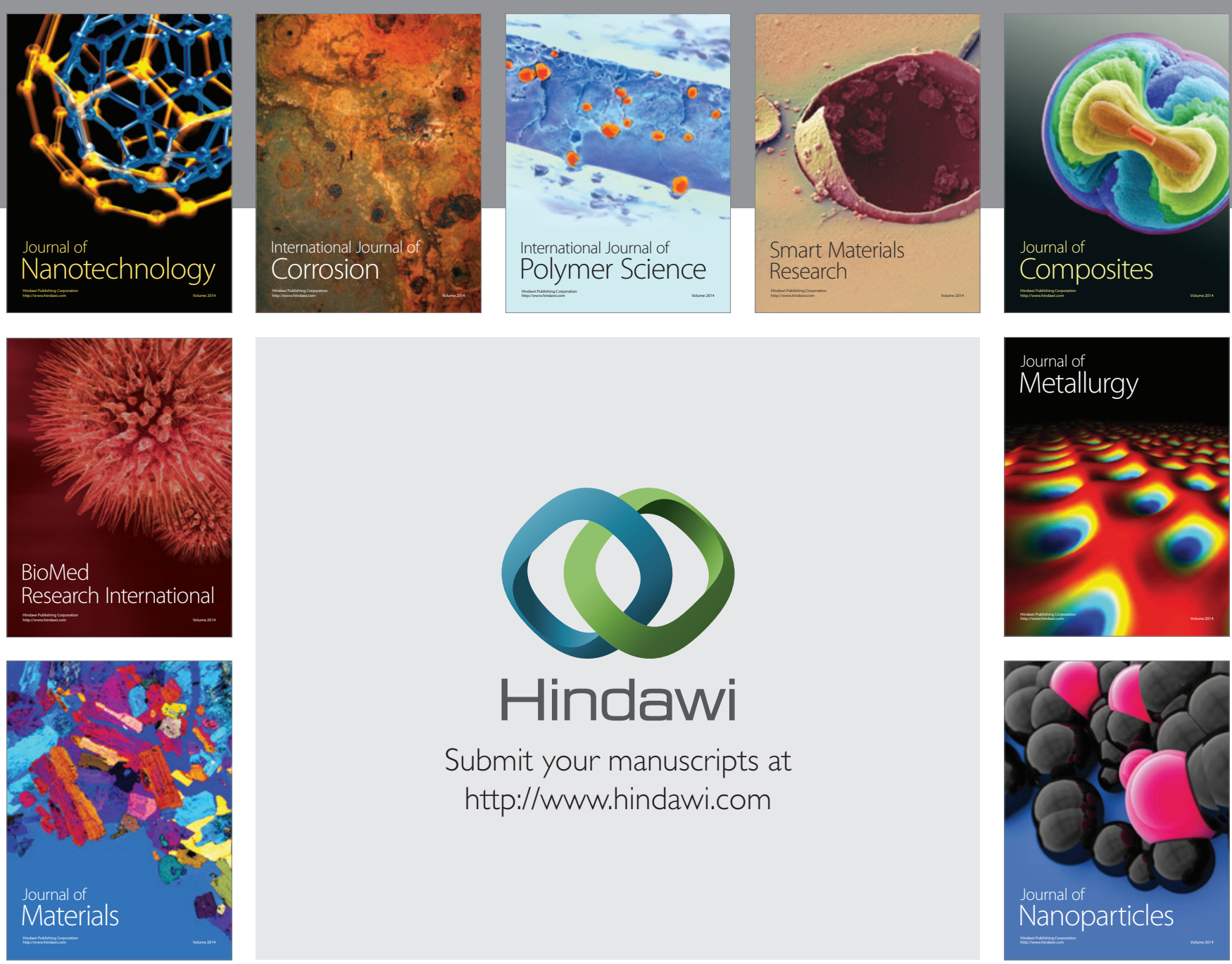

Submit your manuscripts at http://www.hindawi.com
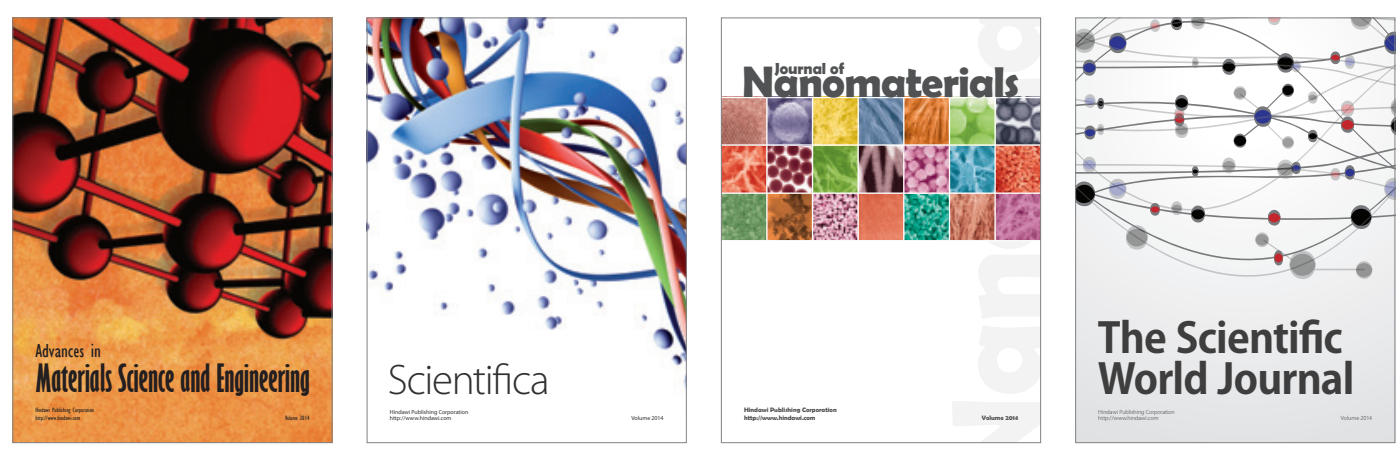

\section{The Scientific World Journal}
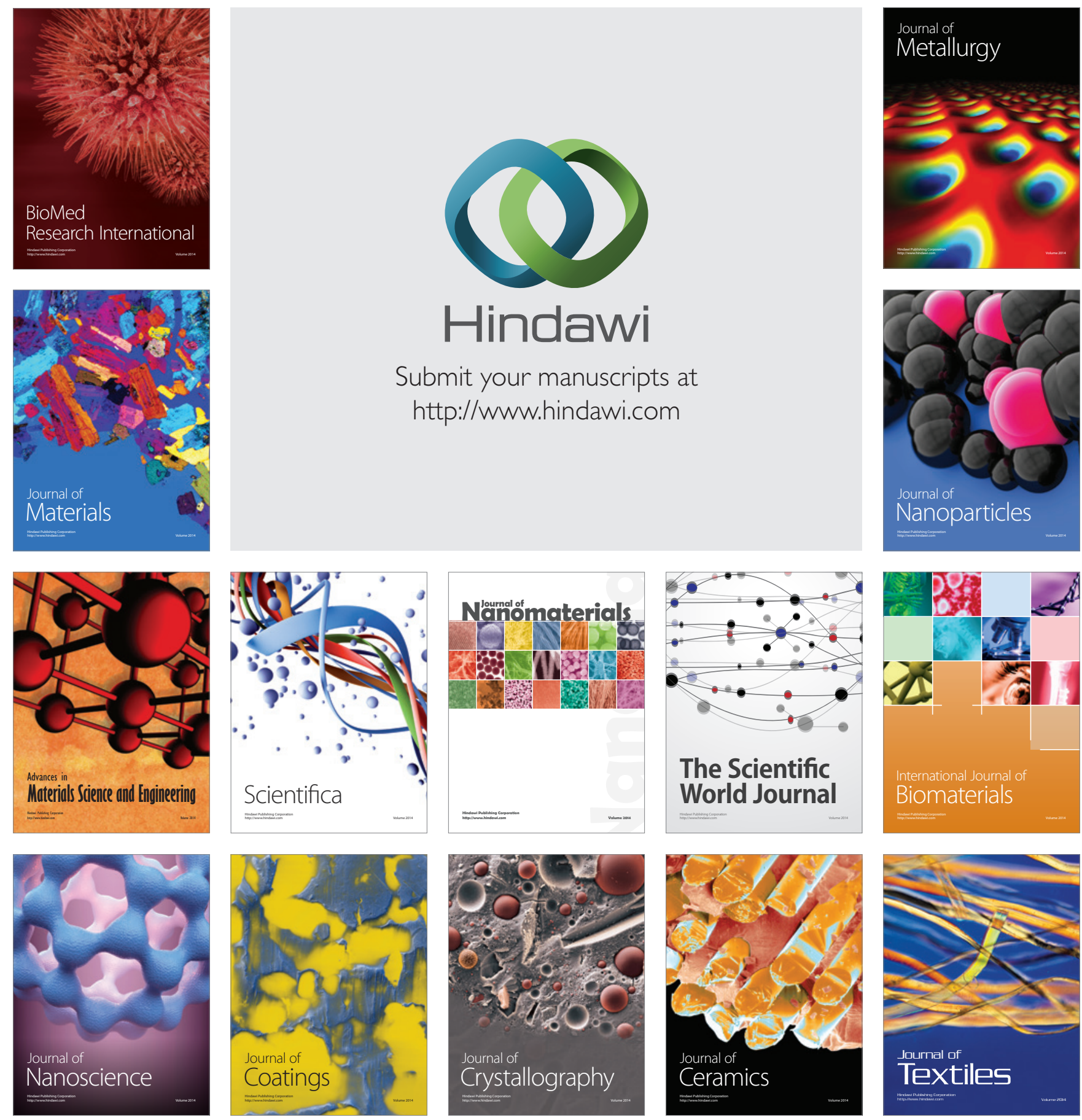